\title{
INVARIANT MANIFOLDS FOR SOME AUTONOMOUS SYSTEMS $\left({ }^{1}\right)$
}

\author{
BY \\ F. S. VAN VLECK(2)
}

1. Introduction. Consider the real differential system

$$
d x / d t=f(x, \mu)
$$

where $\mu$ is a real parameter, $x$ is an $n$-vector with components $x^{i}$, and $f(x, \mu)$ is a real $n$-dimensional vector valued function of $x$ and $\mu$ with components $f^{i}(x, \mu)$. Assume that the system has a periodic solution $x=\phi(t)$ for all $\mu$ and that all the characteristic exponents of $\left(\mathscr{S}_{n}\right)$ based on $\phi$ have zero real parts for $\mu=\mu_{0}$. Following Poincaré we call such a value of $\mu$ a bifurcation value for the system. Under certain restrictions we show that an invariant $(n-1)$-manifold in the $x$-space bifurcates (branches off) from the fixed periodic solutions for values of $\mu$ near $\mu_{0}$. This process will be known as bifurcation from a periodic solution. Poincaré [10] has shown that in the plane such behavior may occur.

Various other authors have studied manifolds defined by solutions of ordinary differential equations. However, most of this work is concerned with the perturbation problem. For an extensive bibliography on this topic see W. T. Kyner [6]. The basic theorem of N. Levinson [9] established the existence of an invariant manifold based on $x=\phi(t)$ in the extended phase space. This manifold was topologically a torus, that is, $\prod_{1}^{n} S^{1}=S^{1} \times S^{1} \times \cdots \times S^{1}\left(S^{n}\right.$ is the unit $n$-sphere) in the $(x, t)$-space. It was assumed that the real parts of $n-1$ of the characteristic exponents based on $\phi(t)$ were negative for all values of the parameter $\mu$. This theorem has been generalized by various authors including Hufford [4], Kyner [5; 7], Diliberto and Hufford [3], and Diliberto [1; 2]. The assumption of stability (the real parts of $n-1$ of the characteristic exponents are negative) has been replaced by the assumption of conditional stability (the real parts of $n-1$ of the characteristic exponents are nonzero). These results differ from ours in three respects. The first is that our invariant manifold lies in the $x$-space;

Presented to the Society September 1, 1960 under the title Bifurcation of an invariant manifold from a periodic solution of a differential system; received by the editors September 14, 1961 and, in revised form, April 9, 1962.

(1) This is in part the author's doctoral dissertation, directed by Professor Lawrence Markus at the University of Minnesota.

(2) This work was supported in part by the Office of Ordnance Research under OOR contract No. DA-11-022-ORD-3369. 
the perturbation problem leads to a manifold in the $(x, t)$-space. Secondly, we show the existence of a manifold which is topologically $S^{n-2} \times S^{1}$; the invariant manifold in the perturbation problem is topologically $\prod_{1}^{n} S^{1}$. The last difference is the nature of the assumptions made concerning the characteristic; exponents.

The technique used to establish our results consists of first constructing a transversal (see Lefschetz [8]) to the periodic solution $x=\phi(t)$ of $\left(\mathscr{S}_{n}\right)$ at any point $\bar{x}=\phi(\bar{t})$ on the trajectory. Then we consider the mapping of this transversal into itself which is defined by following a solution of $\left(\mathscr{S}_{n}\right)$ starting at a point $x$ on the transversal until it strikes the transversal again at a point $y$. For points of the transversal near $\bar{x}$ this defines a transformation $T_{\mu}$ of the transversal into itself which has the fixed point $\bar{x}$ corresponding to the periodic solution $x=\phi(t)$. It is shown that under certain conditions there is an $(n-2)$-dimensional manifold $M_{\mu}$, not containing $\bar{x}$, embedded in the transversal which is left invariant by the transformation $T_{\mu}$. This is done in $\S 2$ by applying the Schauder fixed point theorem to conditionally compact, convex subsets of the Banach spaces of $C^{0}$ and $C^{1}$ functions defined on the compact differentiable manifold $S^{n-2}$. This shows the existence of an invariant $(n-1)$-dimensional manifold for the system $\left(\mathscr{S}_{n}\right)$. This manifold is topologically the product of the $(n-2)$-dimensiona manifold $M_{\mu}$ and the one sphere $S^{1}$. In fact, $M_{\mu}$ is shown to be topologically equivalent to the $(n-2)$-sphere $S^{n-2}$.

The mapping $T_{\mu}$ of the transversal into itself is, by the regularity properties of solutions of ordinary differential equations, a homemorphism of a neighborhood of the point $\bar{x}$ into itself. The mapping $T_{\mu}$ will have the form

$$
v=D(\mu) u+F(u, \mu)
$$

after suitable normalization, where $D(\mu)$ is a constant $(n-1)$ by $(n-1)$ real matrix, $u$ is an $(n-1)$-vector, and $F(u, \mu)$ is an $(n-1)$-dimensional vector valued function of higher order in $u$. In $\S 2$ we show that after placing suitable restrictions on $D(\mu)$ and $F(u, \mu)$ there exists an invariant $(n-2)$-manifold which is topologically $S^{n-2}$ and which contains $u=0(x=\bar{x})$ in its interior.

The characteristic roots of $D(\mu)$ are known to be the characteristic multipliers (other than the one equal to unity) of the variational system of $\left(\mathscr{S}_{n}\right)$ based on the periodic solution $\phi(t)$ (see Lefschetz [8]). Thus conditions imposed on $D(\mu)$ are relatively easy to ascertain. It is, unfortunately, much more difficult to verify if conditions imposed on $F(u, \mu)$ are satisfied by the transversal map.

In order to fix the above ideas a little more firmly in mind we consider the following example.

1.1. EXAmple. Consider the differential system

$$
\begin{aligned}
& x^{\prime}=-y+x(r-1)\left[\mu-\mu(r-1)-(r-1)^{2}\right], \\
& y^{\prime}=x+y(r-1)\left[\mu-\mu(r-1)-(r-1)^{2}\right],
\end{aligned}
$$


where $r^{2}=x^{2}+y^{2}$ and $\mu$ is a real parameter. The right hand side has continuous first derivatives everywhere, and therefore there exists a unique solution through any given noncritical point. The polar equations for $(\mathscr{S})$ are

$$
\begin{aligned}
& r^{\prime}=r(r-1)\left[\mu-\mu(r-1)-(r-1)^{2}\right], \\
& \theta^{\prime}=1 .
\end{aligned}
$$

Since $d r / d t<0$ for $r$ sufficiently large the solutions of $(\mathscr{S})$ are defined for all non-negative time. Also, $(\mathscr{P})$ has $r(t)=1, \theta(t)=t+\theta_{0}$ as a periodic solution for all $\mu$ where $\theta_{0}$ is a constant. For convenience we take $\theta_{0}=0$. Thus any open interval of the positive $x$-axis containing the point $(1,0)$ is transverse to this solution at $(1,0)$.

The variational equation of $(\mathscr{S})$ based on this periodic solution is $(\mathscr{V}): v^{\prime}=A(t) v$ where

$$
A(t)=\left(\begin{array}{lc}
\mu \cos ^{2} t & -1+\frac{1}{2} u \sin 2 t \\
1+\frac{1}{2} u \sin 2 t & \mu \sin ^{2} t
\end{array}\right) .
$$

The characteristic multipliers $\lambda_{1}$ and $\lambda_{2}$ of $(\mathscr{V})$ satisfy

$$
\lambda_{1} \lambda_{2}=\exp \left\{\int_{0}^{2 \pi} \operatorname{Tr}[A(s)] d s\right\}=\exp [2 \mu \pi]
$$

But as it is already known that one of the $\lambda$ 's is equal to unity, say $\lambda_{2}=1$, we have $\lambda_{1}=\exp [2 \mu \pi]$.

In this example every solution starting at a point $(x, 0), x>0$, strikes the positive $x$-axis again after a time $2 \pi$ seconds. Thus the map $T_{\mu}$ has the form

$$
\bar{x}-1=\exp [2 \mu \pi](x-1)-Q(x, \mu),
$$

where $Q$ is of higher order in $(x-1)$. Here $x$ is the $x$-coordinate of the initial value of the solution of $(\mathscr{S})$ starting at $(x, 0)$ at time $t=0$, and $\bar{x}$ is the position on the $x$-axis of the solution at time $t=2 \pi$. If we let $\omega=\exp [2 \mu \pi]-1$, then the linear terms of $T_{\mu}$ are exactly of the form discussed in Theorem 2.5 of $\S 2$. However, it is not obvious that the nonlinear terms are of the desired form.

Let $\rho=r-1$ in the equation for $r^{\prime}$ as given in $(\mathscr{P})$. Then

$$
\rho^{\prime}=\mu \rho-\rho\left[(1+\mu) \rho^{2}+\rho^{3}\right], \quad \theta^{\prime}=1
$$

give the new system. The solution $\rho(t, u, \mu)$ which starts at $u=x-1$ at time $t=0$ satisfies the integral equation

$$
\rho(t, u, \mu)=u e^{\mu t} \exp \left\{\int_{0}^{t}-\left[(1+\mu) \rho^{2}(\tau, u, \mu)+\rho^{3}(\tau, u, \mu)\right] d \tau\right\}
$$


From this it is not difficult to show that

$$
Q(u, \mu)=u e^{2 \mu \pi}\left\{1-\exp \int_{0}^{2 \pi}-\left[(1+\mu) \rho^{2}(\tau)+\rho^{3}(\tau)\right] d \tau\right\}
$$

satisfies the conditions of Theorem 2.5 (or the conditions of the remark following Theorem 2.5).

The above argument shows that there is a 0 -sphere in the transversal which is left invariant by $T_{\mu}$, that is, in this case, there is an invariant point on either side of $x=1, y=0$ for small positive values of $\mu$. Thus there are two periodic solutions branching off from the periodic solution $r(t)=1, \theta(t)=t$ for small positive $\mu$ and, moreover, these both approach $r=1, \theta=t$ as $\mu$ approaches zero.

2. Invariant manifolds. We consider here the problem of finding manifolds which are left invariant by a transformation.

Problem. Let the mapping

$$
T_{\mu}: y=D(\mu) x-Q(x, \mu)
$$

be a homeomorphism of a neighborhood $V$ of the origin in $R^{n+1}$ into $R^{n+1}$. $D(\mu)$ is an $(n+1)$ by $(n+1)$ matrix defined for all $\mu \in\left[0, \mu_{0}\right]$ and $Q(x, \mu)$ is a vector valued function defined on $V \times\left[0, \mu_{0}\right]$. We wish to find conditions which imply the existence of a smooth surface $S_{\mu}$ having the properties:

$\left(\mathrm{P}_{1}\right) S_{\mu}$ is homeomorphic to $S^{n}$,

$\left(\mathrm{P}_{2}\right) S_{\mu}$ is convex with respect to the origin,

$\left(\mathrm{P}_{3}\right) \quad S_{\mu}$ is left invariant by $T_{\mu}$, that is, $T_{\mu} S_{\mu}=S_{\mu}$.

The considerations will be broken into two parts. In the first we consider the case for any $n$ with very restrictive assumptions on the form of $T_{\mu}$. In the second part we take $n=1$ and obtain an invariant surface under "less" restrictive conditions on the form of $T_{\mu}$.

\section{General CONSIDERATIONS, Lipschitz SURFACES.}

Assumptions. It is assumed that $D(\mu)=(1+\mu) A(\mu)$ where $A(\mu)$ is an orthogonal matrix for all $\mu \in\left[0, \mu_{0}\right]$, and $Q(x, \mu)$ satisfies the following conditions.

$\left(\alpha_{1}\right) \quad Q(x, \mu)=P(x, \mu) A(\mu) x$ where $P$ is a real valued function of $x$ and $\mu$ on $V \times\left[0, \mu_{0}\right]$.

$\left(\alpha_{2}\right) \quad 0<P(x, \mu) \leqq M_{0}|x|$ for $0<|x| \leqq r_{0}$, and $0 \leqq \mu \leqq \mu_{0}$.

$\left(\alpha_{3}\right)\left(\partial P / \partial x^{i}\right)(x, \mu)$ is a continuous function of $(x, \mu)$ on $\bar{B}\left(r_{0}\right) \times\left[0, \mu_{0}\right]$ for $i=1,2, \cdots, n+1$. $(\bar{B}(r)$ is the closure of the open ball $B(r)$ or radius $r$ about the origin.)

$\left(\alpha_{4}\right)$ The directional derivative of $P$ along any radial line is positive for each fixed $\mu \in\left[0, \mu_{0}\right]$ and $x \neq 0$, that is, $(\operatorname{grad} P(x, \mu), x)>0$ for $x \neq 0$.

We shall show that the above assumptions imply the existence of a Lipschitz surface $S_{\mu}$ having the properties $\left(\mathrm{P}_{1}\right)-\left(\mathrm{P}_{3}\right)$. However, we first give two examples. 
The first example shows that the condition $\left(\alpha_{1}\right)$ that $Q(x, \mu)$ be factorable is too stringent. Later when we attempt to show the existence of $C^{1}$-surfaces this example will show why some of the hypotheses are needed. The second example is the prototype of transformations $T_{\mu}$ which satisfy the above hypotheses.

2.1. Example. Consider the mapping $T$ defined by

$$
y^{i}=(1+\mu) x^{i}-\left(x^{i}\right)^{3}
$$

where $\left(x^{1}, x^{2}\right)$ are rectangular coordinates in $R^{2}$ and $\mu \geqq 0$. This mapping is a homeomorphism of the open set $V:\left|x^{i}\right|<(1+\mu / 3)^{1 / 2} \quad(i=1,2)$ into itself for $0 \leqq \mu<\frac{1}{2}$. $T$ leaves invariant a unique continuous simple closed curve $S$ in $V$ satisfying properties $\left(\mathbf{P}_{1}\right)-\left(\mathrm{P}_{3}\right) . S$ is, in fact, the square given by $\max \left(\left|x^{1}\right|,\left|x^{2}\right|\right)$ $=\mu^{1 / 2}$. This curve is clearly of class $C^{0}$ and also satisfies a Lipschitz condition. However, the factorization hypothesis $\left(\alpha_{1}\right)$ is not satisfied.

2.2. Example. (Prototype). Let $A(\mu)=I$, the identity matrix, and let $Q(x, \mu)=x|x|^{2}$. Then $P(x, \mu)=|x|^{2}$, and $(\operatorname{grad} P(x, \mu), x)=2|x|^{2}$. Thus the hypotheses $\left(\alpha_{1}\right)-\left(\alpha_{4}\right)$ are clearly satisfied.

2.3. LemMA. Let $T_{\mu}$ be defined as above and suppose $\left(\alpha_{1}\right)-\left(\alpha_{3}\right)$ are satisfied. Then there exists a positive number $\mu_{1}, 0<\mu_{1} \leqq \mu_{0}$, such that for a fixed $\mu \in\left(0, \mu_{1}\right)$ there are two numbers $r_{1}(\mu)$ and $r_{2}(\mu)$ having the properties:

(i) $0<r_{1} \leqq r_{2} \leqq r_{0}$ where $\bar{B}\left(r_{0}\right) \subset V$;

(ii) $r_{1}(\mu), r_{2}(\mu) \rightarrow 0$ as $\mu \rightarrow 0$;

(iii) the annulus $A_{\mu}: r_{1}(\mu) \leqq|x| \leqq r_{2}(\mu)$ is mapped into itself by the mapping $T_{\mu}$.

Proof. Since $0<P(x, \mu) \leqq M_{0}|x|$ for $|x| \neq 0$, assume $r_{0}$ has been chosen sufficiently small that $P(x, \mu)<\frac{1}{2}$ in $\bar{B}\left(r_{0}\right)$.

Let $p_{1}(r, \mu)=\inf _{|x|=r} P(x, \mu)$ and $p_{2}(r, \mu)=\sup _{|x|=r} P(x, \mu)$ for $x \in \bar{B}\left(r_{0}\right)$. Then

$$
|x|\left[1+\mu-p_{2}(|x|, \mu)\right] \leqq|y| \leqq|x|\left[1+\mu-p_{1}(|x|, \mu)\right] .
$$

Also, it can be shown that

$$
\begin{aligned}
\inf _{|x|=r}(\operatorname{grad} P(x, \mu), x /|x|) & \leqq D p_{1}(r, \mu), D p_{2}(r, \mu) \\
& \leqq \sup _{|x|=r}(\operatorname{grad} P(x, \mu), x /|x|)
\end{aligned}
$$

where $D f$ stands for any one of the four derivates of a given function $f$. Thus $D\left[r\left(1+\mu-p_{i}(r, \mu)\right)\right]=1-O(r)$ as $r \rightarrow 0$ uniformly in $\mu$. Hence, for $r$ sufficiently small, $1-O(r)>0$ and, as a consequence of the fact that a function having a positive derived number is strictly increasing, the functions $r\left[1+\mu-p_{i}(r, \mu)\right]$ are strictly increasing. So we need to choose $r_{1}(\mu)$ and $r_{2}(\mu)$ such that $r_{2}\left[1+\mu-p_{1}\left(r_{2}, \mu\right)\right] \leqq r_{2}$ and $r_{1}\left[1+\mu-p_{2}\left(r_{1}, \mu\right)\right] \geqq r_{1}$. We choose these such that we have equality, that is, such that $p_{1}\left(r_{2}, \mu\right)=\mu$ and $p_{2}\left(r_{1}, \mu\right)=\mu$. Let $\bar{\mu}_{0}=\min \left[p_{1}\left(r_{0}, \mu\right): \mu \in\left[0, \mu_{0}\right]\right]$. Then $\bar{\mu}_{0}$ is positive. Let $\mu_{1}=\min \left(\mu_{0}, \bar{\mu}_{0}\right)$. For 
fixed $\mu \in\left(0, \mu_{1}\right]$ consider $p_{1}(r, \mu)-\mu$ as a function of $r$. Now $p_{1}(0, \mu)-\mu=-\mu<0$, and $p_{1}\left(r_{0}, \mu\right)-\mu \geqq \mu_{1}-\mu \geqq 0$. Thus there is a point $r^{\prime}$ such that $p_{1}\left(r^{\prime}, \mu\right)=\mu$. Let $r_{2}=\inf \left[r^{\prime}: p_{1}\left(r^{\prime}, \mu\right)=\mu\right]$. Then $p_{1}\left(r_{2}, \mu\right)=\mu$ and $0<r_{2} \leqq r_{0}$. Since $p_{1}\left(r_{2}, \mu\right) \leqq p_{2}\left(r_{2}, \mu\right)$ we may, in a similar manner, choose $r_{1}, 0<r_{1} \leqq r_{2}$, such that $p_{2}\left(r_{1}, \mu\right)=\mu$.

We next consider the Banach space $C^{0}\left(S^{n}\right)$ of continuous functions defined on $S^{n}$ with the uniform norm. Let $A_{\mu}$ denote the invariant annulus of Lemma 2.3. Let

$$
\mathscr{F}(\mu)=\left[f \in C^{0}\left(S^{n}\right): r_{1}(\mu) \leqq f \leqq r_{2}(\mu)\right] .
$$

The map $T_{\mu}$ induces a transformation of $\mathscr{F}(\mu)$ into itself as follows.

(i) There is a 1-1 correspondence between $\mathscr{F}(\mu)$ and the family of closed $C^{0}$ surfaces lying in $A_{\mu}$ which are convex with respect to the origin. If $f \in \mathscr{F}(\mu)$ and $u \in S^{n}$, then $x^{i}=f(u) u^{i}(i=1,2, \cdots, n)$ gives the corresponding point on $S(f)$, the surface associated with the function $f$.

(ii) $T_{\mu}$ maps $S(f)$ into another surface $T_{\mu} S(f)$ lying in $A_{\mu} . T_{\mu} S(f)$ is a $C^{0}$ surface given parametrically by $v \in S^{n}$ as

$$
y(v)=f(u) A(\mu) u[(1+\mu)-P(f(u) u, \mu)]
$$

where $v=A(\mu) u$.

(iii) The surface $T_{\mu}(S(f))$ given above corresponds to the $C^{0}$ function $g(v)$ defined on $S^{n}$ by

$$
g(v)=f\left(A^{-1}(\mu) v\right)\left[(1+\mu)-P\left(f\left(A^{-1}(\mu) v\right) A^{-1}(\mu) v, \mu\right)\right] .
$$

Thus the mapping $T_{\mu}$ induces a mapping $\bar{T}_{\mu}: f(u) \rightarrow g(v)$ of $\mathscr{F}(\mu)$ into itself.

By hypothesis $\left(\alpha_{4}\right),(\operatorname{grad} P(x, \mu), x)>0$ for $x_{i} \neq 0$. Thus there is a constant $K_{1}$ such that

$$
(\operatorname{grad} P(x, \mu), x) \geqq K_{1}|x| \text { for } x \in A_{\mu} .
$$

Also, we may assume without loss of generality that

$$
r_{0}|\operatorname{grad} P(x, \mu)|<\frac{1}{2} \text { for }(x, \mu) \in \bar{B}\left(r_{0}\right) \times\left[0, \mu_{0}\right] .
$$

2.4. LemmA. There exists a constant $K_{2}$ such that $\left|f\left(u_{1}\right)-f\left(u_{2}\right)\right| \leqq K_{2}\left|u_{1}-u_{2}\right|$ implies that the image function $g(v)=\bar{T}_{\mu} f(u)$ satisfies $\left|g\left(v_{1}\right)-g\left(v_{2}\right)\right| \leqq K_{2}\left|v_{1}-v_{2}\right|$ for $f \in \mathscr{F}(\mu)$.

Proof. This follows directly from the mean value theorem and the preceding assumptions with $K_{2} \geqq\left(r_{2}\right)^{2} / 2 K_{1} r_{1} r_{0}$.

We now choose a subset $\mathscr{F}\left(\mu, K_{2}\right)$ of $\mathscr{F}(\mu)$ defined by $\mathscr{F}\left(\mu, K_{2}\right)=[f \in \mathscr{F}(\mu): f$ satisfies a uniform Lipschitz condition with Lipschitz constant less than or equal to $\left.K_{2}\right]$. We have just shown that $\mathscr{F}\left(\mu, K_{2}\right)$ is left invariant by $\bar{T}_{\mu}$. Moreover, this set is a compact, convex subset of $C^{0}\left(S^{n}\right)$. Now apply the Schauder fixed 
point theorem and obtain the existence of a member $f_{\mu}$ of $\mathscr{F}\left(\mu, K_{2}\right)$ such that $\bar{T}_{\mu} f_{\mu}(u)=f_{\mu}(v)$. Now returning to the mapping $\bar{T}_{\mu}$ we see that the surface $S\left(f_{\mu}\right)$ is invariant under $T_{\mu}$ as a point set.

We may summarize with the following theorem.

\subsection{THEOREM. Let the mapping}

$$
T_{\mu}: y=(1+\mu) A(\mu) x-Q(x, \mu)
$$

be a homeomorphism of a neighborhood $V$ of the origin in $R^{n+1}$ into $R^{n+1}$. Suppose $A(\mu)$ is an orthogonal matrix and that $Q(x, \mu)$ satisfies the conditions $\left(\alpha_{1}\right)-\left(\alpha_{4}\right)$. Then for $\mu>0$ sufficiently small there exists a surface $S_{\mu}$ satisfying properties $\left(\mathrm{P}_{1}\right)-\left(\mathrm{P}_{3}\right)$. The surface $S_{\mu}$ is Lipschitz continuous and $S_{\mu} \rightarrow 0$ as $\mu \rightarrow 0$.

It should be noted that this theorem is also true in case $T_{\mu}$ is a homeomorphism of $R^{1}$ into itself. This can be seen by noting that the annulus which is mapped into itself is just two line segments, one on the negative $x$-axis, the other on the positive $x$-axis. Also, $\operatorname{sgn} T_{\mu}(x)=\operatorname{sgn} x$, so each segment is mapped in a continuous manner into itself by $T_{\mu}$. Thus by the Brouwer fixed point theorem there is an invariant point in each segment, that is, there is an invariant 0-sphere $S^{0}$ contained in the annulus. It also should be noted that in this case the assumption $\left(\alpha_{4}\right)$ is not used. Also, the factorability condition $\left(\alpha_{1}\right)$ is no longer essential to the proof. $x Q(x, \mu)$ positive for all $\mu$ and nonzero $x$ together with $\partial Q / \partial x$ being of first order in $x$ will suffice to establish the existence of the annulus.

\section{II. $C^{1}$ SURFACES FOR MAPPINGS OF $R^{2}$ INTO $R^{2}$.}

Let $T_{\mu}$ be a homeomorphism of a neighborhood $V$ of the origin in $R^{2}$ into $R^{2}$ mapping the polar coordinates $(\bar{\rho}, \bar{\theta})$ into $(\rho, \theta)$ where

$$
\begin{aligned}
& \bar{\rho}=(1+\mu) \rho-g(\rho, \theta, \mu), \\
& \bar{\theta}=\theta+\beta+w(\rho, \theta, \mu) \quad(\beta=\text { constant }) .
\end{aligned}
$$

Let $I_{\theta}$ denote the interval $0 \leqq \theta \leqq 2 \pi, I_{\rho}$ the half open interval $0<\rho \leqq \rho_{0}$, and $I_{\mu}$ the interval $0 \leqq \mu \leqq \mu_{0}$. We make the following assumptions on $g$ and $w$.

$\left(\alpha_{5}\right) \quad g$ and $w$ are periodic of period $2 \pi$ in $\theta$, and are continuous on $\bar{I}_{\rho} \times I_{\theta} \times I_{\mu} . \partial g / \partial \rho, \partial g / \partial \theta, \partial w / \partial \rho, \partial w / \partial \theta$ are continuous on $I_{\rho} \times I_{\theta} \times I_{\mu}$.

$\left(\alpha_{6}\right) \quad 0<g(\rho, \theta, \mu) \leqq M_{0} \rho^{2}, 0<\partial g / \partial \rho \leqq M_{1} \rho,|w| \leqq M_{2} \rho$, and $|\partial w / \partial \rho| \leqq M_{3}$ uniformly in $\theta$ and $\mu$ on $I_{\rho} \times I_{\theta} \times I_{\mu}$.

$\left(\alpha_{7}\right)$ The first partial derivatives of $g$ and $w$ are zero for $\rho=0$, and satisfy a uniform Lipschitz condition in $(\rho, \theta)$ with constant $L$ on $I_{\rho} \times I_{\theta} \times I_{\mu}$ ( $L$ is independent of $\mu$ ).

$\left(\alpha_{8}\right) \quad g / \rho^{2}$ is a nondecreasing function of $\rho>0$ for each fixed $\theta$ and $\mu$.

$\left(\alpha_{9}\right)$ For $\rho \in \bar{I}_{\rho}$ define $G(\rho, \mu)=\sup _{\theta} g(p, \theta, \mu)$ and $g(\rho, \mu)=\inf _{\theta} g(\rho, \theta, \mu)$. Assume $\rho^{2}|\partial g / \partial \theta| \leqq g^{2}(\rho, \mu) / 3 C$ and $\rho|\partial w / \partial \theta| \leqq g(\rho, \mu) B / C$ where 


$$
C=\max \left[16 M_{0}, 48\left(M_{3}+B\right)\right]
$$

and $B \geqq 1$ is a fixed constant.

Hypotheses $\left(\alpha_{5}\right)-\left(\alpha_{7}\right)$ give the order and regularity conditions imposed on $g$ and $w$. These are seen to be stronger than those imposed in Part I. However, the factorability assumption $\left(\alpha_{1}\right)$ has been deleted. $\left(\alpha_{8}\right)$ is the direct analogue of $\left(\alpha_{4}\right)$, and $\left(\alpha_{9}\right)$ gives conditions which may be interpreted as circulatory conditions on $g$ and $w$.

We next reconsider the two previous examples. The first indicates the nature of hypothesis $\left(\alpha_{9}\right)$ and shows that some hypothesis of this nature is needed to show the existence of an invariant $C^{1}$ surface. The second is a prototype.

2.6. Example. Consider the mapping $T$ defined by

$$
y^{i}=(1+\mu) x^{i}-\left(x^{i}\right)^{3}
$$

of the neighborhood $V$ of the origin $R^{2}$ into itself. We have previously shown that in $V$ this transformation has a unique $C^{0}$ curve $S$ which is invariant under $T$ and which has properties $\left(P_{1}\right)-\left(P_{3}\right) . S$ was clearly not of class $C^{1}$. Simple calculations show that $T$ satisfies hypotheses $\left(\alpha_{5}\right)-\left(\alpha_{8}\right)$, but not $\left(\alpha_{9}\right)$.

2.7. EXAMPLE. Let $\bar{\rho}=(1+\mu) \rho-\rho^{3}, \bar{\theta}=\theta$. Then it follows directly that $\left(\alpha_{5}\right)-\left(\alpha_{9}\right)$ are satisfied since $\partial g / \partial \theta=0$ and $\partial w / \partial \theta=0$.

We now proceed to show the existence of an invariant $C^{1}$ surface having the desired properties. As before, the Schauder fixed point theorem is applied to an appropriate subset of a Banach space. We begin by stating the following lemma which is analogous to Lemma 2.3.

2.8. Lemma. Let $T_{\mu}$ be defined as above, and suppose $\left(\alpha_{5}\right),\left(\alpha_{6}\right)$ and $\left(\alpha_{8}\right)$ are satisfied. Then there exists a positive number $\mu_{1}, 0<\mu_{1} \leqq \mu_{0}$ such that for $\mu \in\left(0, \mu_{1}\right]$ there are numbers $\rho_{1}(\mu)$ and $\rho_{2}(\mu)$ having properties (i)-(iii) of Lemma 2.3. In fact, $\rho_{1}$ and $\rho_{2}$ can be chosen so that $\mu \rho_{2}=g\left(\rho_{2}, \mu\right)$ and $\mu \rho_{1}=G\left(\rho_{1}, \mu\right)$.

Let $\mathscr{B}$ be the class of all $C^{1}$ functions $\rho(\theta)$ such that $\rho(\theta+2 \pi)=\rho(\theta)$ with the usual $C^{1}$ norm:

$$
\|\rho\|=\sup |\rho(\theta)|+\sup |d \rho / d \theta| .
$$

Let $R_{\mu}=\left\{\rho \in \mathscr{B}: \rho_{1}(\mu) \leqq \rho(\theta) \leqq \rho_{2}(\mu), \theta \in I_{\theta}\right\}$ for $\mu \in\left(0, \mu_{1}\right]$.

2.9. Lemma. If hypotheses $\left(\alpha_{5}\right),\left(\alpha_{6}\right)$ and $\left(\alpha_{9}\right)$ are satisfied, then there is a number $\rho_{3}, 0<\rho_{3} \leqq \rho_{0}$, such that for $\rho(\theta) \in \mathscr{B}, 0<\rho(\theta) \leqq \rho_{3}$, and $|d \rho / d \theta|$ sufficiently small the image curve $\bar{\rho}=\bar{\rho}(\bar{\theta}) \in R_{\mu}$.

Proof. Since $\bar{\theta}=\theta+\beta+w(\rho, \theta, \mu)$ and $\rho=\rho(\theta), \bar{\theta}=f(\theta)=\theta+\alpha+w(\rho(\theta), \theta, \mu)$ and $d \bar{\theta} / d \theta=1+(\partial w / \partial \rho)(d \rho / d \theta)+\partial w / \partial \theta$. Thus, for $d \rho / d \theta$ and $\rho$ sufficiently small, we have $d \bar{\theta} / d \theta>0$, and hence, by the implicit function theorem, we may 
solve for $\theta$ as a function of $\bar{\theta}$. Denote this function by $\theta=\theta(\bar{\theta})$. Then we have $\bar{\rho}=\bar{\rho}(\bar{\theta})=(1+\mu) \rho(\theta(\bar{\theta}))-g(\rho(\theta(\bar{\theta})), \theta(\bar{\theta}), \mu)$. That $\bar{\rho}(\bar{\theta})$ is periodic of period $2 \pi$ follows from the periodicity of $\rho(\theta), g(\rho, \theta, \mu)$, and $w(\rho, \theta, \mu)$. Since $\theta(\bar{\theta})$ is of class $C^{1}, \bar{\rho}(\bar{\theta})$ is of class $C^{1}$. That $\bar{\rho} \in R_{\mu}$ follows from Lemma 2.8 .

2.10. Lemma. Suppose $\left(\alpha_{5}\right),\left(\alpha_{6}\right)$ and $\left(\alpha_{9}\right)$ are satisfied. Then for $\mu \in\left(0, \mu_{1}\right]$ and $\rho \in R_{\mu}$ the inequality $|d \rho / d \theta| \leqq \mu / C$ implies $|d \bar{\rho} / d \bar{\theta}| \leqq \mu / C$.

Proof. Without loss of generality we may assume $\mu_{0} \leqq 1, \rho_{0} \leqq 1, \rho_{3}=\rho_{0}$ ( $\rho_{3}$ is as given in Lemma 2.9), and $\partial g / \partial \rho \leqq 1$. Also, by the choice of $C,|d \rho / d \theta|$ is so small that Lemma 2.9 is applicable.

Let $\mu \in\left(0, \mu_{1}\right]$ and $\rho \in R_{\mu}$. Then the image curve $\bar{\rho}(\bar{\theta}) \in R_{\mu}$ and

$$
d \bar{\rho} / d \bar{\theta}=(d \theta / d \bar{\theta})\{(d \rho / d \theta)[1+\mu-\partial g / \partial \rho]-\partial g / \partial \theta\} .
$$

Since $g / \rho^{2}$ is an increasing function of $\rho$,

$$
\partial g / \partial \rho \geqq 2 g(\rho, \mu) / \rho \geqq 2 \mu g\left(\rho_{1}, \mu\right) / G\left(\rho_{1}, \mu\right) .
$$

Also, by the mean value theorem, $\left(\alpha_{6}\right)$ and $\left(\alpha_{9}\right)$,

$$
G(\rho, \mu)-g(\rho, \mu) \leqq 2 \pi g^{2}(\rho, \mu) / 3 C \rho^{2},
$$

and hence $g(\rho, \mu) / G(\rho, \mu) \geqq 6 / 7$. Using this, the preceding estimate for $\partial g / \partial \rho$, $|d \rho / d \theta| \leqq \mu / C$ and $\left(\alpha_{9}\right)$,

$$
\begin{aligned}
\mid(d \rho / d \theta)[1+ & \mu-\partial g / \partial \rho]-\partial w / \partial \theta \mid \\
& \leqq(\mu / C)[1+\mu-12 \mu / 7]+g^{2}(\rho, \mu) / 3 C \rho^{2} \\
& \leqq(\mu / C)(1-5 \mu / 7)+\mu^{2} / 3 C \leqq(\mu / C)(1-8 \mu / 21) .
\end{aligned}
$$

Next, $d \bar{\theta} / d \theta=1+(d \rho / d \theta)(\partial w / \partial \rho)+\partial w / \partial \theta$, and thus

$$
\begin{aligned}
|d \bar{\theta} / d \theta-1| & \leqq M_{3} \mu / C+g(\rho, \mu) B / \rho C \\
& \leqq(\mu / C)\left(M_{3}+B\right) .
\end{aligned}
$$

From this we have $|d \theta / d \bar{\theta}| \leqq 1+\mu / 24$, and finally,

$$
|d \bar{\rho} / d \bar{\theta}| \leqq(1+\mu / 24)(\mu / C)(1-8 \mu / 21) \leqq \mu / C .
$$

For $\mu \in\left(0, \mu_{1}\right]$, let

$$
S_{\mu}(K)=\left\{\rho \in R_{\mu}:\left|(d \rho / d \theta)\left(\theta_{1}\right)-(d \rho / d \theta)\left(\theta_{2}\right)\right| \leqq K\left|\theta_{1}-\theta_{2}\right|, \theta_{1}, \theta_{2} \in I_{\theta}\right\} .
$$

Since this set is a compact, convex subset of $\mathscr{B}$, we need only show that there is some $K$ for which $S_{\mu}(K)$ is mapped into itself by $T_{\mu}$ in order to be able to use the Schauder fixed point theorem.

2.11. Lemma. Suppose $\left(\alpha_{5}\right)-\left(\alpha_{9}\right)$ are satisfied and $\mu$ and $\rho$ have been chosen 
as in Lemmas 2.8-2.10. Then there exists a $K$ such that $T_{\mu}$ maps $S_{\mu}(K)$ into itself.

Proof. Let $\rho^{\prime}=d \rho / d \theta$ and $\bar{\rho}^{\prime}=d \bar{\rho} / d \bar{\theta}$. Also, let $\delta=\mid \bar{\rho}^{\prime}\left(\bar{\theta}_{1}\right)-\bar{\rho}^{\prime}\left(\bar{\theta}_{2}\right)$ where $\bar{\theta}_{i}=\theta_{i}+\beta+w\left(\rho\left(\theta_{i}\right), \theta_{i}, \mu\right)(i=1,2)$. For convenience, let $\bar{\theta}=f(\theta), d \bar{\theta} / d \theta=f^{\prime}(\theta)$, and

$$
F(\theta)=d \rho / d \theta[1+\mu-(\partial g / \partial \rho)(\rho(\theta), \theta, \mu)]-(\partial g / \partial \theta)(\rho(\theta), \theta, \mu)
$$

Then

and

$$
\bar{\delta}=\left[f^{\prime}\left(\theta_{1}\right)\right]^{-1}\left\{\left[F\left(\theta_{1}\right)-F\left(\theta_{2}\right)\right]+\bar{\rho}^{\prime}\left(\bar{\theta}_{2}\right)\left[f^{\prime}\left(\theta_{2}\right)-f^{\prime}\left(\theta_{1}\right)\right]\right\}
$$

$$
\begin{aligned}
|\bar{\delta}| & \leqq(1+\mu / 24)\left\{\mid\left(F\left(\theta_{1}\right)-F\left(\theta_{2}\right)|+(\mu / C)| f^{\prime}\left(\theta_{2}\right)-f^{\prime}\left(\theta_{1}\right) \mid\right\}\right. \\
& \leqq(1+\mu / 24)\left\{\left|F\left(\theta_{1}\right)-F\left(\theta_{2}\right)\right|+(\mu / C)\left(2 L+M_{3} K\right)\left|\theta_{1}-\theta_{2}\right|\right\}
\end{aligned}
$$

since, by $\left(\alpha_{6}\right)$ and $\left(\alpha_{7}\right)$,

$$
\left|f^{\prime}\left(\theta_{2}\right)-f^{\prime}\left(\theta_{1}\right)\right| \leqq\left(2 L+M_{3} K\right)\left|\theta_{1}-\theta_{2}\right|
$$

if $|\delta| \leqq K\left|\theta_{1}-\theta_{2}\right|$. Also, using estimates as in the proof of Lemma 2.10 and using $\left(\alpha_{7}\right)$,

$$
\begin{aligned}
\left|F\left(\theta_{1}\right)-F\left(\theta_{2}\right)\right| \leqq & |\delta|(1-5 \mu / 7)+(\mu / C) L\left\{\left|\rho\left(\theta_{1}\right)-\rho\left(\theta_{2}\right)\right|+\left|\theta_{1}-\theta_{2}\right|\right\} \\
& +L\left\{\left|\rho\left(\theta_{1}\right)-\rho\left(\theta_{2}\right)\right|+\left|\theta_{1}-\theta_{2}\right|\right\} \\
\leqq & \left|\theta_{1}-\theta_{2}\right|\left\{K(1-5 \mu / 7)+L(1+\mu / C)^{2}\right\} .
\end{aligned}
$$

Now choose $K$ so large that $-5 K_{\mu} / 7+L(1+\mu / C)^{2} \leqq-\mu K / 6$. Then,

$$
|\bar{\delta}| \leqq(1+\mu / 24)\left\{K(1-\mu / 6)+(\mu / C)\left(2 L+M_{3} K\right)\right\}\left|\theta_{1}-\theta_{2}\right| .
$$

If, in addition to the above restriction on $K$, we require that $K \geqq 12\left(2 L+M_{3} K\right) / C$, then

$$
|\bar{\delta}| \leqq K(1-\mu / 48)\left|\theta_{1}-\theta_{2}\right| .
$$

Next, by the mean value theorem and $\left(\alpha_{9}\right)$,

$$
\begin{aligned}
\left|\bar{\theta}_{1}-\bar{\theta}_{2}\right| & \geqq\left|\theta_{1}-\theta_{2}\right|\left(1-\mu M_{3} / C-\mu B / C\right) \\
& \geqq\left|\theta_{1}-\theta_{2}\right|(1-\mu / 48) .
\end{aligned}
$$

Combining these, $|\bar{\delta}| \leqq K\left|\bar{\theta}_{1}-\bar{\theta}_{2}\right|$.

We may summarize by stating the following theorem.

2.12. TheOREM. Let $T_{\mu}$ be defined as above, mapping $(\rho, \theta)$ into $(\bar{\rho}, \bar{\theta})$, and satisfy assumptions $\left(\alpha_{5}\right)-\left(\alpha_{9}\right)$. Then for sufficiently small $\mu>0$ there exists a curve $C_{\mu}$ of class $C^{1}$ which satisfies properties $\left(\mathrm{P}_{1}\right)-\left(\mathrm{P}_{3}\right)$. If $C_{\mu}$ is given by $\rho=\rho(\theta)$, then $d \rho / d \theta$ is Lipschitz continuous. Also, $C_{\mu} \rightarrow 0$ as $\mu \rightarrow 0$. 


\section{BIBLIOGRAPHY}

1. S. P. Diliberto, Perturbation theory of periodic surfaces. I, Tech. Rep. No. 24, Dept. of Math., Univ. of Calif., Berkeley, Calif., April, 1958.

2. - Perturbation theory of periodic surfaces. II, Tech. Rep. No. 9, Dept. of Math., Univ. of Calif., Berkeley, Calif., April, 1957.

3. S. P. Diliberto and G. Hufford, Perturbation theorems for nonlinear ordinary differential equations, Contributions to the Theory of Nonlinear Oscillations, Vol. III, pp. 207-236, Princeton Univ. Press, Princeton, N. J., 1956.

4. G. Hufford, Banach spaces and the perturbation of ordinary differential equations, Contributions to the Theory of Nonlinear Oscillations, Vol. III, pp. 173-195, Princeton Univ. Press, Princeton, N. J., 1956.

5. W. T. Kyner, A fixed point theorem, Contributions to the Theory of Nonlinear Oscillations, Vol. III, pp. 197-205, Princeton Univ. Press, Princeton, N. J., 1956.

6. - Invariant manifolds, Univ. of Southern Calif. Rep., Los Angeles, Calif., 1959.

7. - Small periodic perturbations of an autonomous system of vector equations, Contributions to the Theory of Nonlinear Oscillations, Vol. IV, Princeton Univ. Press, Princeton, N. J., 1958.

8. S. Lefschetz, Differential equations: geometric theory, Interscience, New York, 1957.

9. N. Levinson, Small periodic perturbations of an autonomous system with a stable orbit, Ann. of Math. (2) 52 (1950), 727-738.

10. H. Poincaré, Les méthodes nouvelles de la méchanique céleste, Dover,New York, 1957.

UNIVERSITY OF MINNESOTA,

MinNEAPOLIS, MinNESOTA

MassachusetTs Institute of Technology,

CAMBRIDGE, MassachusetTS 